Ann. Biol. anim. Bioch. Biophys., 1977, 17 (5 B), 887-895.

\title{
La cétogenèse chez le ruminant à jeun : influence des acides gras volatils, du glucose et de l'alanine
}

\author{
par C. DEMIGNÉ, C. REMESY
}

Station de Physiopathologie de la Nutrition, I.N.R.A., Theix, Saint Genès Champanelle, 63110 Beaumont.

Summary. Ketogenesis in the fosting ruminant. Effect of volatile fatty acids, glucose and olanine.

Lambs weighing about $30 \mathrm{~kg}$ were fasted 48 or $78 \mathrm{~h}$ to study the effect of volatile fatty acids (AGV) and glucogenic substrates on ketogenesis. The AGV were administered intraruminaly and the glucose and alanine in $L$ or DL form subcutaneously.

Excess acetate had an antilipolytic effect accompanied by a slight rise in ketone bodies, while excess butyrate was antilipolytic and ketogenic. The propionate was very antiketogenic for free fatty acids or for butyrate. L-alanine had little effect, whereas its antiketogenic action was strong but brief; this effect did not seem due solely to hyperglycemia. DL-alanine had effects similar to L-alanine, but they occurred later.

The mechanisms of liver ketogenesis regulation are discussed in relation to acyl CoA transport to the mitochondria or to the orientation of acefyl CoA metabolism towards lipogenesis rather than towards ketogenesis; particular attention is given to butyrate.

\section{Introduction.}

Dans certaines conditions physiologiques particulières telles que la gestation ou la lactation, le ruminant est particulièrement exposé aux cétoses, pour deux raisons principales : la carence en substrats glucoformateurs et l'abondance de composés potentiellement cétoformateurs. L'équilibre entre ces deux catégories de substrats est complexe puisqu'il dépend de la ration et de la situation physiologique (drainage de composés glucoformateurs par les foetus ou vers la lactation). Ce travail a pour but d'étudier l'orientation du métabolisme des acides gras libres (AGL) et du butyrate $\left(C_{4}\right)$ vers la cétogenèse, ainsi que l'action du glucose, du propionate $\left(C_{3}\right)$ et de l'alanine. Le modèle utilisé sera l'agneau d'environ $30 \mathrm{~kg}$ à jeun. Ce modèle permet d'obtenir des lipomobilisations importantes, par contre les besoins en composés glucoformateurs sont évidemment inférieurs à ceux de brebis en fin de gestation.

\section{Matériel et méthodes.}

- Animaux. Des agneaux de race Charmoise ou Limousine d'un poids d'environ $30 \mathrm{~kg}$ ont reçu pendant les 3 semaines précédant l'expérience un régime comportant du foin et du concentré ad libitum afin de permettre une lipogenèse maximale. Pour l'expérience les animaux ont été placés à jeun 48 ou 72 heures. 
Les acides gras volatils $(A G V)\left(C_{2}, C_{3}, C_{4}\right)$ ont été administrés par injection intraruminale (aiguille pour injection spinale $18 \mathrm{G} 3 \mathrm{BD}$ ) sous forme de solution $2 \mathrm{M}$ amenées à $\mathrm{pH}$ 5,5 par $\mathrm{NaOH}$. Le glucose et l'alanine (formes $\mathrm{L}$ ou $\mathrm{DL}$ neutralisées à $\mathrm{pH} 7$ par $\mathrm{NaOH}$ ) ont été injectés par voie sous-cutanée à la dose de $0,5 \mathrm{~g} / \mathrm{kg}$. Le sang a été prélevé dans la veine jugulaire et immédiatement centrifugé sur héparine.

- Mesures biochimiques. Les dosages enzymatiques sur plasma sont effectués après déprotéinisation par $\mathrm{HClO}_{4} 0,6 \mathrm{M}(2 \mathrm{v})$ et les techniques de dosage du glucose, d'acétoacétate, de 3 hydroxybutyrate $\left(3-\mathrm{OHC}_{4}\right)$, de L-alanine ainsi que des AGL ont été décrites par ailleurs (Rémésy et Demigné, 1976). Les AGV ont été dosés par chromatographie en phase gazeuse après déprotéinisation éthanolique (Rémésy et Demigné, 1974).

\section{Résultats.}

- Réponse au jeûne (łabl. 1). La glycémie chez le jeune ruminant est relativement élevée. Le jeûne s'accompagne d'une hypoglycémie et d'une diminution des taux d'alanine et d'acétate. Les AGL augmentent ainsi que les corps cétoniques. Les animaux nourris présentaient une légère cétogenèse, sans doute à partir du $C_{4}$ absorbé. L'hypercétonémie du jeune est modérée, comparée à celle des brebis cétosiques (3 à $4 \mathrm{mM}$ ) pour une même lipomobilisation.

\section{TABLEAU 1}

Réponse au jeane de paramètres plasmatiques de la néoglucogenèse et de la cétogenèse chez des agneaux de $30 \mathrm{~kg}$ environ, nourris avec un régime foin + concentré ad libitum depuis trois semaines.

Les résultats sont les moyennes $\pm \sigma / \sqrt{\mathrm{N}}$

\begin{tabular}{|c|c|c|}
\hline & $\begin{array}{c}\text { Nourris }(10) \\
(\mathrm{mM})\end{array}$ & $\begin{array}{c}\text { A jeûn } 48 \mathrm{~h}(10) \\
(\mathrm{mM})\end{array}$ \\
\hline $\begin{array}{l}\text { Glucose } \ldots \ldots \ldots \ldots \ldots \ldots \ldots \\
\text { Alanine } \ldots \ldots \ldots \ldots \ldots \ldots \\
\text { AGL } \ldots \ldots \ldots \ldots \ldots \ldots \\
\text { 3-hydroxybutyrate } \ldots \ldots \ldots \ldots \\
\text { Acétoacétate } \ldots \ldots \ldots \ldots \ldots \ldots \\
\text { Acétate } \ldots \ldots \ldots \ldots \ldots \ldots\end{array}$ & $\begin{array}{l}4,11 \pm 0,19 \\
0,25 \pm 0,03 \\
0,33 \pm 0,04 \\
0,25 \pm 0,02 \\
0,44 \pm 0,06\end{array}$ & $\begin{array}{l}3,52 \pm 0,05 \\
0,14 \pm 0,06 \\
1,00 \pm 0,17 \\
0,57 \pm 0,03 \\
0,18 \pm 0,03 \\
0,14 \pm 0,01\end{array}$ \\
\hline
\end{tabular}

- Effets de l'acétate (fig. 1 et 2). Les surcharges en $C_{2}$ s'accompagnent d'une très forte élévation des taux périphériques (plus de $1,5 \mathrm{mM}$ ) en raison de la faible captation hépatique de cet acide. Le $C_{2}$ à fortes concentrations semble légèrement hypoglycémiant, alors qu'il est nettement antilipolytique. Le 3-OHC ${ }_{4}$ augmente mais de façon très iardive par rapport au pic de $\mathrm{C}_{2}$ dans le sang.

- Effets du butyrate (fig. 2 et 3). Les surcharges ne provoquent pas d'élévation sensible de cet acide dans le sang périphérique (fig. 2). Le foie, à la différence du $C_{2}$, capte la presque totalité du $C_{4}$ absorbé qui n'a pas été transformé dans la paroi du rumen. On évite ainsi les réponses de caractère extraphysiologique constatées lorsque des concentrations anormales en $C_{4}$ sont présentes dans le sang systémique. Dans ces conditions on observe une légère tendance à l'hypoglycémie, probablement due à une 
insulino-sécrétion. Le $C_{4}$ manifeste un effet antilipolytique très net, sans qu'il soit possible de préciser s'il s'agit d'un effet direct ou indirect (par l'intermédiaire du 3-OHC $\mathrm{H}_{4}$ ). La cétogenèse observée provient principalement du $C_{4}$ puisque la captation hépatique des AGL tend à être diminuée, cette captation étant proportionnelle à la concentration des AGL dans le sang afférent au foie (Soler-Argilaga, Infante, Polonovski, 1973). II n'est toutefois pas possible de préciser la part de la cétogenèse effectuée par la paroi du rumen par rapport à celle du foie.
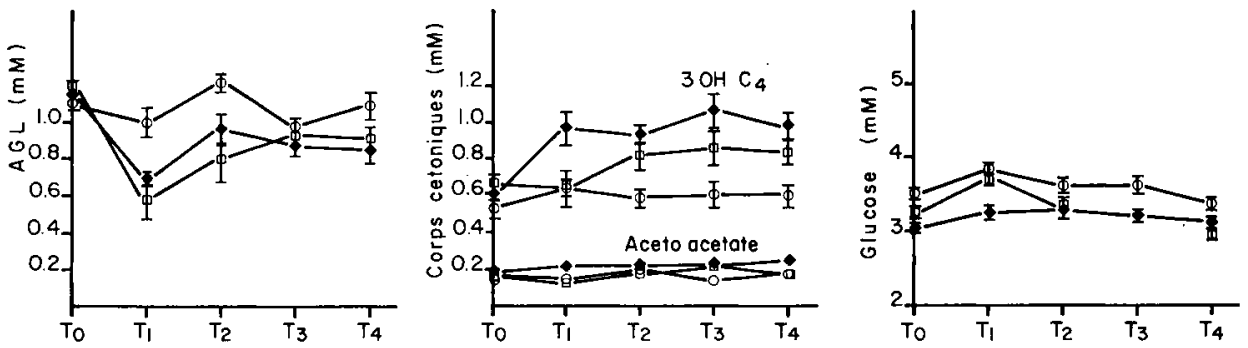

FIG. 1. - Effets de surcharges à pH 5,5 en acide acétique $(1 \mathrm{~g} / \mathrm{kg})$ et butyrique $(0,5 \mathrm{~g} / \mathrm{kg})$ administrés dans le rumen en deux fois à $T 0$ ef $T 1$ sur les concentrations plosmatiques en glucose, AGL ef corps cétoniques chez des agneaux de 26 à $34 \mathrm{~kg}$ à jeun $48 \mathrm{~h}$.

Témoins (0).

Surcharge acide acétique (a).

Surcharge acide butyrique ( $\$$ ).

Chaque point expérimental est la moyenne $\pm \sigma / \sqrt{N}(N=4)$.

$T_{0}, T_{1}, T_{3} \ldots$. heures

- Effets d'une surcharge en glucose (fig. 4). La surcharge provoque une hyperglycémie très importante qui s'accompagne d'un effet antilipolytique relativement modéré, alors que l'effet anticétogène est très net et prolongé.

- Effets de surcharge en propionate (fig. 2 et 3). De même que celles en $C_{4}$, ces
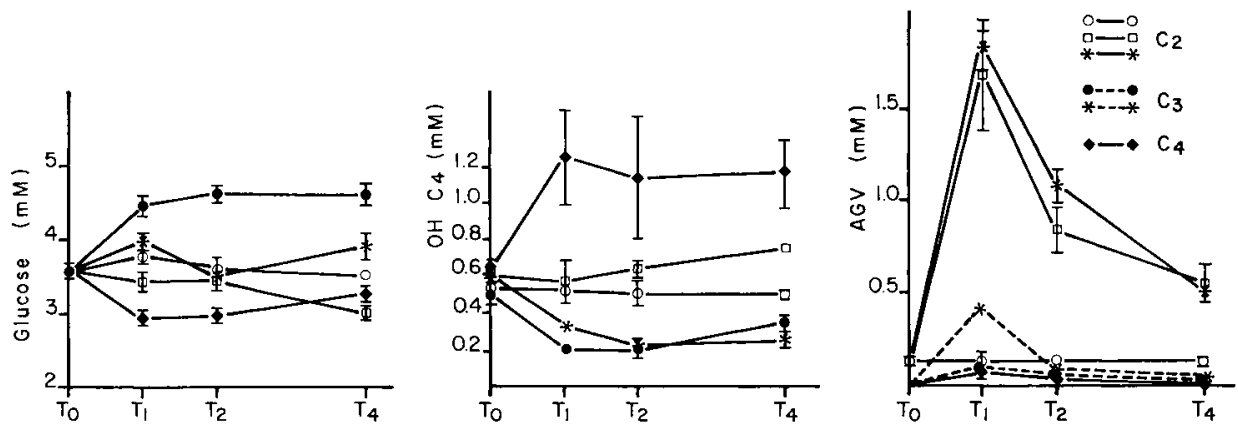

FIG. 2. - Effets de fortes surcharges à pH 5,5 en acide acétique $(2 \mathrm{~g} / \mathrm{kg})$, propionique et butyrique $(1 \mathrm{~g} / \mathrm{kg})$ ainsi qu'en acide acétique +- propionique administrés dans le rumen à TO sur les concentrations plasmatiques en AGV, glucose et 3 hydroxybutyrafe chez des agneaux de 20 d̀ $28 \mathrm{~kg}$ d jeun $48 \mathrm{~h}$.

Témoins (o).

Surcharge acide acétique (a).

Surcharge acide propionique (•).

Surcharge acide butyrique $(\$)$.

Surcharge acides acétique + propionique $(*)$.

Chaque point expérimental est la moyenne $\pm \sigma / \sqrt{N}(N=4)$. 
surcharges ne provoquent pas d'élévation importante des taux périphériques de $C_{3}$, sauf lorsqu'il est associé à un autre AGV. Il semble alors exister un phénomène de compétition entre AGV pour la captation hépatique.
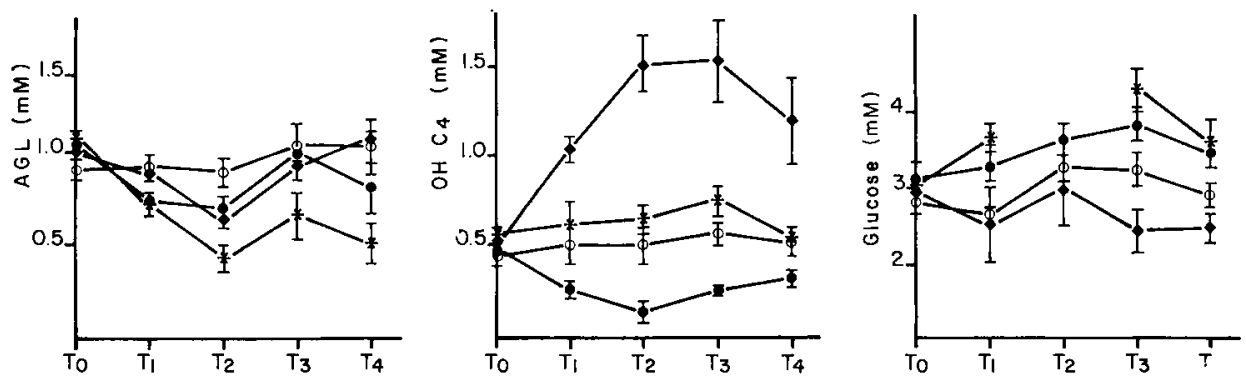

FIG. 3. - Effets de surcharges ò pH 5,5 en acide propionique, butyrique $(1 \mathrm{~g} / \mathrm{kg})$ ou en acide propionique + bufyrique $(1 \mathrm{~g} / \mathrm{kg}$ pour chaque acide) administrés dans le rumen à T0 et T1 sur les concentrations plasmatiques en glucose, AGL et 3 hydroxybutyrate chez des agneaux de 26-34 kg à jeun $48 \mathrm{~h}$.

Témoins (0).

Surcharge acide propionique (๑).

Surcharge acide butyrique $(\bullet)$.

Surcharge acide propionique + butyrique $(*)$.

Chaque point expérimental est la moyenne $\pm \sigma / \sqrt{N}(N=4)$.
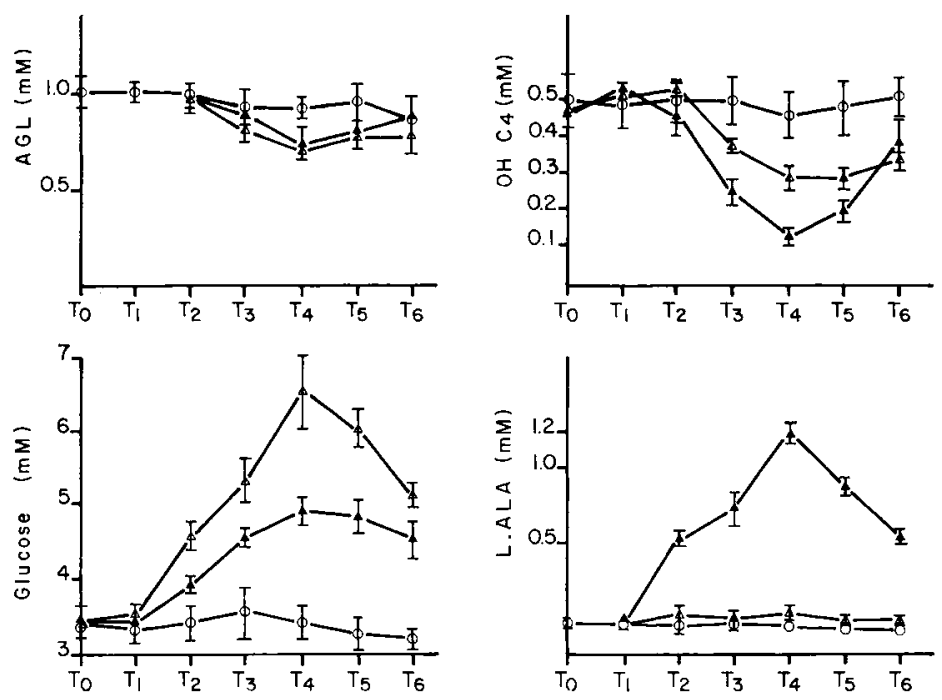

FIG. 4. - Effets de surcharges en glucose ef L-alanine $(0,5 \mathrm{~g} / \mathrm{kg})$ administrés par voie sous cutanée d̀ $T 1$, $T 2$ ef T3 sur les concentrations plasmatiques en glucose, L-alanine, AGL et 3 hydroxybutyrafe chez des agneaux de $26-34 \mathrm{~kg}$ ò jeun $48 \mathrm{~h}$.

Témoins (o).

Surcharge glucose $(\Delta)$.

Surcharge L-alanine (A).

Chaque point expérimental est la moyenne $\pm \sigma / \sqrt{\mathrm{N}}(\mathrm{N}=4)$. 
Le $C_{3}$ est hyperglycémiant et antilipolytique. Ces effets s'accompagnent d'une activité anticétogène durable, même lorsque les AGL sont assez peu diminués. L'action exercée par le $C_{3}$ sur la cétogenèse hépatique ne semble pas entièrement due à l'hyperglycémie. Associé au $C_{4}$, le $C_{3}$ (fig. 3) est aussi hyperglycémiant. L'effet antilipolytique des deux AGV s'additionne apparemment, par l'élévation de la glycémie due au $C_{3}$ ou par une élévation de l'insuline induite par le $C_{3}$ ou le $C_{4}$, en raison d'une captation hépatique incomplète. L'action cétogène du $C_{4}$ est presque totalement abolie par le $C_{3}$ (Waldo et Schultz, 1960).

- Effets de la $L$ et de la DL-alanine. La L-alanine possède une action hyperglycémiante très voisine de celle de la DL-alanine (fig. 5), toutefois inférieure à celle du glucose d̀ dose équivalente (fig. 4). La L-alanine manifeste une action antilipolytique variable et assez brève, alors que la DL-alanine diminue les AGL plus tardivement. La L-alanine est très anticétogène mais de façon peu durable, l'effet tend à disparaître alors que la glycémie reste élevée ; on peut même parfois observer un « effet rebond 》 en fin d'expérience. La DL-alanine agit moins rapidement mais son effet est ensuite plus prolongé, la diminution du $3-\mathrm{OHC}_{4}$ est comparable à celle enregistrée avec la L-alanine.
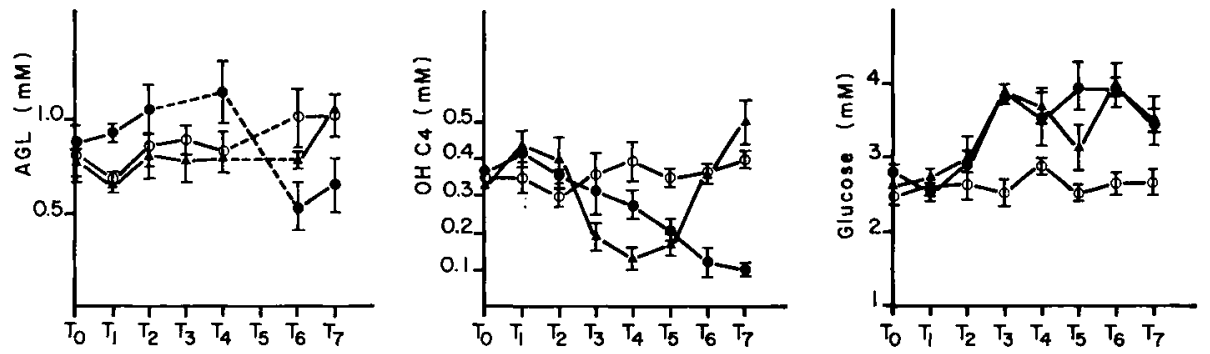

FIG. 5. - Effets de surcharges en L-alanine ef DL-alanine $(0,5 \mathrm{~g} / \mathrm{kg})$ administrés par voie sous-cutanée à T0, T1 et T2 sur les concentrations plasmatiques en glucose, AGL ef 3 hydroxybutyrate chez des agneaux de 32 à $38 \mathrm{~kg}$ à jeun $72 \mathrm{~h}$.

Témoins (O).

Surcharge L-alanine (A).

Surcharge DL-alanine (•).

Chaque point expérimental est la moyenne $\pm \sigma / \sqrt{N}(N=4)$.

\section{Discussion.}

L'administration des AGV dans le rumen permet d'étudier dans des conditions relativement physiologiques l'apport de ces composés au niveau du foie. Le métabolisme du rumen affecte en particulier le $\mathrm{C}_{4}$ qui est transformé en partie en $3-\mathrm{OHC}_{4}$ : ce processus serait toutefois limité par les capacités de la 3-hydroxy-3-méthyl-glutaryl (HMG) CoA synthétase du rumen (Baird et al., 1970), si bien que la cétogenèse ruminale augmenterait assez peu en cas de surcharges en $C_{4}$ (Weigand, Young, Mc Gilliard, 1972). Ces données proviennent d'animaux nourris, et on connaît mal l'impact des modifications métaboliques du jeûne sur cette cétogenèse ruminale. Nous n'observons qu'un effet global, et, lors de surcharges en $C_{3}+C_{4}$, il est possible que le $C_{3}$ (ou le glucose) conduise à une utilisation hépatique prépondérante du $C_{4}$ en diminuant la cétogenèse ruminale. Selon Menahan, Schultz et Hoekstra (1966), c'est dans les condi- 
tions d'hypoglycémie de la fin de la gestation que la transformation du $C_{4}$ au niveau du rumen est maximale.

Le métabolisme du $\mathrm{C}_{3}$ en lactate ef pyruvate par la paroi du rumen est peu important (Weekes, 1972) contrairement aux résultats de Bergman et Wolff (1971) ; quanł aux voies d'utilisation du $\mathrm{C}_{2}$ (production du $\mathrm{CO}_{2}$ ou de lipides), elles sont nécessairement très limitées en cas de surcharges chez un animal à jeûn. Le pouvoir cétogène du $\mathrm{C}_{2}$ est habituellement considéré comme négligeable (faible captation hépatique, utilisation au niveau du cycle de Krebs ou vers la lipogenèse). Le $C_{2}$ est pourtant un métabolite extrêmement diffusible et le foie renferme une acétate thiokinase mitochondriale, faible il est vrai devant l'acétyl CoA hydrolase cytoplasmique (Quraishi et Cook, 1972). Cette situation explique l'élévation de l'acétate plasmatique, d'origine endogène, chez des animaux ayant une forte cétogenèse (Remesy et Demigné, 1976). Par ailleurs, le $C_{3}$ ef le $C_{4}$ inhiberaient l'utilisation mitochondriale du $C_{2}$ (Smith, 1971 ; Ash et Baird, 1973) ; toutefois le $C_{2}$ est potentiellement cétogène chez l'animal en déficit énergétique lorsque les activités du cycle de Krebs et de la lipogenèse sont insuffisantes pour utiliser l'acétate activé. L'élévation des corps cétoniques enregistrée tardivement avec la surcharge en $\mathrm{C}_{2}$ ne correspond pas nécessairement à une cétogenèse à partir de cet acide ; en effet il peut exister une compétition avec les corps cétoniques au niveau de l'utilisation périphérique.

L'utilisation du $\mathrm{C}_{4}$ au niveau du foie est favorisée par l'existence d'une acyl CoA synthéthase mitochondriale très active et la possibilité de pénétration directe dans la mitochondrie sans l'intermédiaire du système carnitine acyltransférase qui constituerait le site primaire de régulation du métabolisme des AGL (Mc Garry et Foster, 1974, 1976). De plus, alors que la cétogenèse à partir des AGL doit obligatoirement passer par le stade acétyl CoA, celle à partir du $C_{4}$ peut théoriquement se limiter à une dégradation jusqu'au stade acétoacétyl CoA (selon Huth et al., 1973 ; 60 p. 100 de la cétogenèse à partir du $\mathrm{C}_{4}$ ). Cette particularité permet à la cétogenèse d'échapper à la rétroinhibition exercée sur la thiolase par l'acétoacétyl-CoA (Huth ef al., 1975). L'élévation des corps cétoniques provoquée par le $C_{4}$ semble indépendante de la quantité des AGL disponible chez le Rat (Williamson ef al., 1974), ce point reste à vérifier chez le Ruminant qui présente dans ces conditions une diminution du taux des AGL. L'action antilipolytique du $C_{4}$ peut s'exercer au niveau du tissu adipeux (Metz, Lopes-Cardozo et Van Den Bergh, 1974) ou par effet insulinosécréteur (Manns ef Boda, 1967). Ces deux actions supposent le passage de $C_{4}$ dans la circulation générale ; selon Manns, Boda et Willes (1967) des teneurs aussi faibles que $0,03 \mathrm{mM}$ en butyrate dans le sang périphérique correspondent à une insulino-sécrétion appréciable. L'effet antilipolytique du $\mathrm{C}_{4}$ pourrait s'exercer aussi par l'intermédiaire du 3-OHC (Goberna ef al., 1974) toutefois la sensibilité du pancréas endocrine du Ruminant à ce stimulus est certainement inférieure à celle au $C_{4}$ et est controversée (Horino et al., 1968.)

Le propionate est le précurseur glucoformateur le mieux capté par le foie et le plus anticétogène. L'effet anticétogène s'exerce par l'intermédiaire de la baisse de captation des AGL et par l'orientation du métabolisme des AGL captés vers la réestérification ou l'oxydation. De plus, le propionyl CoA semble posséder une action inhibitrice sur l'HMG CoA synthéłase mitochondriale (Bush et Milligan, 1971). L'action inhibitrice du $C_{3}$ sur la cétogenèse à partir du $C_{4}$ peut s'expliquer aussi par l'activation 
du cycle de Krebs, favorisée par la diminution du taux des AGL. L'inhibition de la cétogenèse est très forte et il est peu probable que tout le $C_{4}$ capté soit transformé en $\mathrm{CO}_{2}$. La seule voie d'utilisation de l'acétyl CoA reste la lipogenèse. Si la lipogenèse hépatique est faible chez le ruminant pour l'acétate (Bauman ef Davis, 1975), il en est certainement autrement pour le butyrate et les acides gras moyens.

D'après Treacher, Baird et Young (1976) l'effet anticétogène du glucose passe par la diminution de la disponibilité en AGL pour la $\beta$ oxydation hépatique mais aussi par l'élévation de l'oxaloacétate disponible pour la condensation avec l'acétyl CoA.

La L-alanine exerce une action anticétogène apparemment supérieure à celle du glucose, bien que l'effet antilipolytique soit assez voisin et relativement modéré. La L-alanine stimule la sécrétion de glucagon et peut ainsi élever indirectement celle d'insuline (Muller, Aoki et Cahill, 1975). Selon Brockman (1976) l'effet potentiellement cétogénique ef lipolytique du glucagon serait limité par l'insulino-sécrétion qui l'accompagne. L'action de la L-alanine ne se limite certainement pas à la stimulation de la sécrétion de glucagon ou d'insuline : Mc Garry et Foster (1974) attribuent aux substrats glucoformateurs une action inhibitrice sur l'acylcarnitine transférase. En outre, l'alanine stimulerait la néoglucogenèse en inhibant la pyruvate kinase hépatique (Friedrichs et Schoner, 1974).

Genuth ef Castro (1974) ont trouvé chez l'homme que l'alanine pouvait faire diminuer le 3-OHC $\mathrm{OH}_{4}$ même si les AGL s'élèvent. Ces résultats, ef les hypoalaninémies observées chez l'enfant ef la brebis cétosiques (Pagliara, Karl et Devivo, 1972 ; Rémésy et Demigné, 1976) indiquent qu'après le propionate, l'alanine est le composé glucoformateur le plus anticétogène. Cette action a été montrée chez le ruminant cétosique par Weik et Zander (1975), cependant ces travaux n'ont porté que sur un nombre de cas très limité. L'utilisation de la L-alanine risque toutefois d'être limitée par le coût élevé de ce produit et la fugacité de l'action antilipolytique et anticétogène. La DL-alanine semble posséder des propriétés sensiblement différentes de la L-alanine, dues au métabolisme plus lent de la $D$-alanine par l'intermédiaire des $D$ amino acide oxydases hépatiques et rénales, par rapport à celui de la L-alanine par les transaminases.

Le mécanisme de l'action anticétogène au niveau hépatique des précurseurs glucoformateurs est certainement différent selon que la cétogenèse s'effectue à partir des $A G L$ ou du $C_{4}$. Dans le premier cas, l'action la plus vraisemblable se situe au niveau de la réestérification sous forme de triglycérides, par l'intermédiaire du système acyl carnitine transférase ; ce mécanisme est d'ailleurs compatible avec les hypothèses avancées par Mc Garry et Foster (1976) qui font intervenir les teneurs hépatiques en glycogène ainsi qu'en carnitine. Dans le second cas, du fait que le $C_{4}$ est obligatoirement activé dans la mitochondrie, il ne reste que la possibilité d'une augmentation de la lipogenèse cytoplasmique favorisée par les précurseurs glucogéniques (apport en NADPH).

Les modalités ef les possibilités de régulation du passage des acétyl CoA vers le cytoplasme sont encore mal connues, que ce soit par les voies classiques (citrate, acétylcarnitine) ou, comme il a été proposé par Rous et Favarger (1973), via l'acétoacétate dont la production mitochondriale à partir du $\mathrm{C}_{4}$ peut subsister.

Réunion Groupe Développement INRA/Productions animales Thiverval-Grignan, 14-15 avril 1976. 


\section{Références}

ASH R., BAIRD G. D., 1973. Activation of volatile fatty acids in bovine liver and rumen epithelium. Evidence for control by autoregulation. Biochem. J., 136, 311-319.

BAIRD G. D., HIBBITT K. G., LEE J., 1970 . Enzymes involved in acetoacetate formation in various bovine tissues. Biochem. J., 117, 703-709.

BAUMAN D. E., DAVIS C. L., 1975. Regulation of lipid metabolism 459-509. In MCDONALD I.W. WARNER I. C., Digestion and metabolism in the ruminant, University of New England, Publ. Unit, Armidal, Australie.

BERGMAN E. N., WOLFF J. E., 1971. Metabolism of volatile fatty acids by liver and portal-drained viscera in sheep. Am. J. Physiol., 221, 586-592.

BROCKMAN R. P., 1976. Effects of glucagon and insulin on lipolysis and kelogenesis in sheep. Can. J. comp. Med., 40, 166-170.

BUSH R. S., MILLIGAN L. P., 1971. Study of the mechanism of inhibition of ketogenesis by propionate in bovine liver. Can. J. anim. Sci, 51, 121-127.

FRIEDRICHS D., SCHONER W., 1974. Regulation of gluconeogenesis by alanine. Biochem. biophys. Acta, 343, 341-355.

GENUTH M., CASTRO J., 1974. Effects of oral alanine on blood betahydroxybutyrate and plasma glucose. Insulin, free fatty acids, and growth hormone in normal and diabetic subjects. Metabolism, 23, 375-385.

GOBERNA R., TAMARIT J., OSORIO R., FUSSGANGER R., TAMARIT J., PFEIFFER E. F. 1974. Action of $\beta$-hydroxybutyrate, acetoacetate and palmitate on the insulin release in the perfused isolated rat pancreas. Horm. Metab. Res., 6, 256-260.

HORINO M., MACHLIN L. J., HERTELENDY F., KIPNIS D. M., 1968. Effect of short-chain fatty acids on plasma insulin in ruminant and non-ruminant species. Endocrinology, 83, 118-128.

HUTH W., DIERICH C., OEYNHAUSEN V., SEUBERT W., 1973. On the mechanism of ketogenesis and its control. I. On a possible role of acetoacetyl CoA thiolase in the control of ketone body production. Hoppe-Seyler's Z. Physiol. Chem., 354, 635-649.

HUTH W., JONAS R., WUNDERLICH I., SEUBERT W., 1975. On the mechanism of ketogenesis and its controls. Purification kinetic mechanism and regulation of different forms of mitochondrial acetoacetyl-CoA thiolases from ox liver. Eur. J. Biochem., 59, 475-489.

Mc GARRY J. D., FOSTER D. W., 1974. The metabolism of (-) Octanoylcarnitine in perfused livers from fed and fasted rats. Evidence for a possible regulatory role of carnitine actyltransferase in the control of ketogenesis. J. biol. Chem., 249, 7984-7990.

Mc GARRY J. D., FOSTER D. W., 1976. Ketogenesis and its regulation. Am. J. Med., 61, 9-13.

MANNS J. G., BODA J. M., 1967. Insulin release by acetate, propionate, butyrate and glucose in lambs and adult sheep. Am. J. Physiol., 212, 747-755.

MANNS J. G., BODA J. M., WILLES R. F., 1967. Probable role of propionate and butyrate in control of insulin secretion in sheep. Am. J. Physiol., 212,, 756-764.

MENAHAN L. A., SCHULTZ L. H., HOEKSTRA W. G., 1966. Factors affecting ketogenesis from butyric acid in the ruminant. J. Dairy Sci., 49, 835-845.

METZ H. M., LOPES-CARDOZO M., VAN DEN BERGH S. G., 1974. Inhibition of lipolysis in bovine adipose tissue by butyrate and $\beta$-hydroxybutyrate. FEBS Letters, 47, 19-23.

MULLER W. A., AOKI T. T., CAHILL G. F., 1975. Effect of alanine and glycine on glucagon secretion in post absorptive and fasting obese man. J. clin. endocrinol. Mełab., 40, 418-425.

PAGLIARA A. S., KARL I. E., DE VIVO D. C., 1972. Hypoalaninemia : a concomitant of ketotic hypoglycemia. J. clin. Invest., 51, 1440-1449.

QURAISHI S., COOK R. M., 1972. Utilization of volatile fatty acids in ruminants. IV. Relative activities of acetyl COA synthetase and acetyl CoA hydrolase in mitochondria and intracellular localization of acetyl CoA synthetase. J. agric. Food Chem., 20, 91-95.

RÉMÉSY C., DEMIGNÉ C., 1974. Determination of volatile fatty acids in plasma after ethanolic extraction. Biochem. J., 141, 85-91.

RÉMESY C. DEMIGNÉ C., 1976.Variations in some plasma metabolites from neoglucogenesis and ketogenesis in pregnant ewes in relation to diet. Ann. Rech. vétér. 7, 329-341. 
ROUS S., FAVARGER P., 1973. The role of acetoacetate in the transfer of acetyl units outside the mitochondria in liver and adipose tissue of rats or mice. FEBS Letters, 32, 231-233.

SMITH R. M., 1971. Interactions of acetate, propionate and butyrate in sheep liver mitochondria. Biochem. J., 124, 877-881.

SOLER-ARGILAGA C., INFANTE R., POLONOVSKI J., 1973. Influence of chain length and degree of unsaturation on plasma free fatty acid uptake by the perfused rat liver. Biochim. biophys. Acta, 326, 167-173.

TREACHER R. J., BAIRD D., YOUNG J. L., 1976. Anti-ketogenic effect of glucose in the lactating cow deprived of food. Biochem. J., 158, 127-134.

WALDO D. R., SCHULTZ L. A., 1960 . Blood and rumen changes following the intraruminal administration of glucogenic materials. J. Dairy Sci., 43, 496-505.

WEEKES T. E. C., 1972. Effect of pregnancy and lactation in sheep on the metabolism of propionate by the ruminal mucosa and on some enzymic activities in the ruminal mucosa. J. Agric. Sci. Camb., 79, 409-421.

WEIGAND E., YOUNG J. W., Mc GILLIARD A. D., 1972. Extent of propionate metabolism during absorption from the bovine ruminoreticulum. Biochem. J., 126, 201-209.

WEIK H., ZANDER H. D., 1975. Der Einflu $\beta$ von Alanin auf die Acetonamie der Milchkuh und auf die phlorizin-induzierte Acetonamie des Schafes. Zbl. Vet. Med., A, 22, 520-523.

WILLIAMSON D. H., ELLINGTON E. V., ILLIC V., SAAL J., 1974. Hepatic effects of satured and unsatured short-chain fatty acids and the control of ketogenesis in vivo, 191-209. In LUNDQUIST F., TYGSTRUP N. Regulation of hepatic metabolism. A. Benzon Symposium VII. Munskgaard, Copenhague. 